\title{
IVERMECTIN AND ONCHOCERCAL OPTIC NEURITIS: SHORT-TERM EFFECTS
}

\author{
I. MURDOCH ${ }^{1.2}$, A. ABIOSE ${ }^{1,3}$, O. BABALOLA ${ }^{1}$, A. BIRD ${ }^{4}$, S. COUSENS ${ }^{5}$, J. EVANS $^{1,2}$ and B. JONES ${ }^{1,2}$ \\ Kaduna, Nigeria and London
}

\begin{abstract}
SUMMARY
In 1982 the macrocyclic lactone, ivermectin, was first tested in human patients with onchocerciasis. It has since undergone phase I to IV trials and is now being widely distributed in onchocercal areas. The previous microfilaricide, diethylcarbamazine citrate (DEC), is known to precipitate or exacerbate active optic neuritis in some onchocercal patients, as part of a wider inflammatory response (the Mazzotti reaction). Ivermectin may also cause a mild reaction, especially in people with high microfilarial loads. Few data are available concerning the effect of ivermectin on active optic neuritis. A large, randomised, double-masked, phase IV trial is reported. Individuals were screened for evidence of optic nerve disease (OND), and those identified as possible cases of OND underwent detailed ophthalmic examination, including fluorescein angiography, before being dosed with ivermectin or placebo. A total of 6831 persons were screened of whom $856(13 \%)$ underwent angiography prior to dosing. At 7-14 days after dosing an attempt was made to reexamine $50 \%$ of adults over the age of 20 years, including all those with OND. Six hundred and eighty-eight repeat or new angiograms were performed. During this period, 5 new cases of active optic neuritis and one case of exacerbation of existing optic neuritis were identified. Five of these individuals had received placebo and one ivermectin. Two individuals with optic neuritis before dosing had improved after 7-14 days. One had received placebo, the other ivermectin. Ivermectin does not appear to precipitate or exacerbate optic neuritis at a period of 7-14 days.
\end{abstract}

Onchocerciasis is identified by the WHO as a major global public health problem and has been included as a target

From: the Kaduna Collaboration for Research on Onchocerciasis. 'Department of Ophthalmology. Ahmadu Bello University Teaching Hospital, Kaduna, Nigeria: International Centre for Eye Health. Institute of Ophthalmology, London, UK; ${ }^{3}$ National Eye Centre Kaduna. Nigeria; ${ }^{4}$ Moorfields Eye Hospital, London. UK: ${ }^{5}$ London School of Hygiene and Tropical Medicine, London, L K.

Correspondence to: Ian Murdoch, Department of Preventive Ophthalmology, Institute of Ophthalmology, Bath Street, London EC1 V 9EL, UK. disease for active research within WHO's Special Programme for Research and Training in Tropical Diseases (TDR). It is estimated that, worldwide, about 18 million individuals are infected with onchocerciasis with a further 68 million people at risk of infection.' The disease affects primarily the skin and eyes and may have a major socioeconomic impact through visual impairment; blindness prevalences exceeding $10 \%$ have been reported in heavily infected populations.

Measures for combating onchocerciasis include use of larvicides in rivers (to control the vector, Simulium damnosum) and drug therapy. Until recently therapy relied on two drugs: diethylcarbamazine citrate (DEC, Heterozan or Banocide) and suramin. Administration of the macrofilaricide suramin requires hospital admission and monitoring since it is given intravenously in multiple weekly doses. It has a reversible nephrotoxicity that is used to monitor dosage and its use in the high doses recommended for onchocerciasis is associated with idiosyncratic reactions including exfoliative dermatitis, diarrhoea, and even death. DEC has been the most frequently used drug in onchocerciasis. It is a microfilaricide that regularly produces a marked eosinophilic reaction (pruritis, and papular skin eruptions). This reaction was first described in Mexico by Mazzotti, who proposed its use as a supplementary diagnostic aid for onchocerciasis." This suggestion was taken up and the drug widely used, for both the diagnosis and the treatment of the disease. For many years the principal objections to the use of DEC were the dermatitis, lymphadenopathy, headache and vertigo it produced. In the late 1970s, however, it was suggested that the use of DEC might also result in impairment of vision, in particular, through the precipitation of optic atrophy. ${ }^{4.5}$ This effect was demonstrated conclusively in 1980 by Bird et al. ${ }^{6}$ who found that treatment with DEC precipitated active optic neuritis in 9 of 18 patients, and worsened pre-existing optic neuritis in a further 3 patients.

The macrocyclic lactone, ivermectin, has been used as an antihelminthic in cattle for many years. In 1982 ivermectin was tested in human patients with onchocerciasis ${ }^{7}$ 
and found to reduce significantly the microfilarial counts in skin snips of infected individuals for periods of 6 months to 1 year. It has since undergone phase I to IV trials and is now being widely distributed to communities at risk of onchocercal infection. Ivermectin may cause a mild pruritis, sometimes with lymphadenopathy, especially in people with high microfilarial loads and, rarely, hypotensive episodes are seen. ${ }^{8}$ As well as the clearing of microfilariae from the skin, reductions in the presence of uveitis, limbitis and punctate keratitis have been reported. ${ }^{9}$ Few data are available concerning the effect of ivermectin on active optic neuritis. The trial reported here was designed to determine whether or not ivermectin precipitates or exacerbates active optic neuritis in the 7-14 day period following dosing. With DEC, optic neuritis is precipitated within 2-3 days. Our clinical experience has shown that the active optic neuritis associated with onchocerciasis lasts from several weeks to 1 year or more. This time period of follow-up was therefore chosen in order to allow sufficient time for the neuritis to develop and not enough time for it to resolve following dosing.

\section{METHODS}

Thirty-four rural communities, in two areas of Kaduna State, Northern Nigeria, were selected for inclusion in the trial. ${ }^{10}$ In these communities the overall prevalence of onchocercal infection (as measured by skin-snip positivity) was $49 \%$ among those aged 5 years and above, and $72 \%$ among those aged 20 years and above. The community microfilarial load was $3.5 \mathrm{mf} / \mathrm{mg}$ skin. The vast majority of people in these communities live as subsistence farmers. In 1988/9 an extensive ophthalmic screening examination was performed at a central location in each village, with the aim of examining all registered individuals according to a structured protocol and identifying all those with definite or suspected optic nerve disease. Individuals were examined by a team of six trained ophthalmic nurses who underwent a 4 week period of training to minimise inter-observer variation. The tests employed included:

Visual acuity: using single E optotypes at $6 \mathrm{~m}$ in available outdoor light, with spectacle correction if routinely worn. Visual fields: Visual fields were assessed with counting fingers in four peripheral quadrants, a $6 \mathrm{~mm}$ white target in the paracentral field, and a test for colour desaturation. Contrast sensitivity: Using the Arden grating, each eye was tested with plates 3 and 5 for the threshold of appreciation of the sinusoidal pattern.

Colour vision: In view of the fact that a majority of the population tested were illiterate, the Birch colour vision test using shape recognition was used. Eight colour plates were used to test eyes for colour discrimination. Threeplates tested red/green discrimination and five plates blue/ yellow discrimination.

Pupil assessment: The nurses employed the swinging light test to determine whether an afferent pupiliary defect was present in either eye.
Optic disc appearance: Provided the ocular media were clear, all discs were assessed by the nurses through undilated pupils for pallor or swelling of the nerve head.

In addition the nurses examined the external eye, cornea, iris and lens.

The normal ranges for the colour vision and contrast sensitivity tests were established in pre-tests. Any person with a pinhole acuity of less than $6 / 9$, a field defect, an abnormal pupil, an abnormal optic disc, or whose colour or contrast sensitivity tests fell in the range of the poorest $5 \%$ of the pre-test population, in either eye, underwent Friedmann field analysis and was referred to one of two ophthalmologists for examination.

The ophthalmologists had a full complement of diagnostic instruments, including slit lamp with lenses and tonometry, and direct and indirect ophthalmoscopes. After a full ophthalmic examination according to a structured protocol, all individuals examined by the ophthalmologist underwent fluorescein angiography, unless they refused such examination or it was contraindicated (by poor general health, pregnancy, young age, etc.).

Partly in order to monitor the sensitivity of the screening examination, a computer-generated random sample of individuals was identified. All those in the random sample underwent examination by the ophthalmologist.

\section{Definition of Optic Nerve Disease (OND)}

A diagnosis of definite OND was made in the presence of marked disc pallor recorded by an ophthalmologist together with defects in visual function and or pupillary light response, or in the presence of active optic neuritis confirmed by fluorescein angiography.

\section{Dosing}

At the time of registration each individual was given a unique identifying number. These numbers were allocated sequentially. At the same time each individual was also allocated to one of 30 'treatment groups' $(1,2 \ldots, 30)$, again sequentially, each individual's 'treatment group' being pre-printed on his or her registration card. Merck, Sharp and Dohme, manufacturers of ivermectin, provided 30 numbered, but otherwise identical, containers. Fifteen of these containers held ivermectin, the other fifteen a placebo. There was no visible difference between the two types of tablet. Merck, Sharp and Dohme allocated ivermectin and placebo to the different containers. Following the completion of the ophthalmic examinations, each individual was weighed to determine how many tablets of ivermectin/placebo should be given. After being given a full explanation of the study in the local language each individual confirmed his or her consent with a thumb-print prior to dosing with tablets from the container corresponding to the treatment group printed on the registration card, carried out under the supervision of a medical doctor. The ophthalmologists and ophthalmic nurses were unaware of who received ivermectin and who received placebo. Ivermectin was given at a dosage of $150 \mu \mathrm{g} / \mathrm{kg}$. 
This trial was approved by the WHO ethics committee and by the local ethics committee in Kaduna.

\section{Follow-up}

Seven to 14 days after dosing the communities were revisited. All compounds were visited and asked about visual problems since dosing. Anyone reporting a problem was re-examined. Individuals who had previously been examined by the ophthalmologist (those with definite or suspected OND and those in the random sample) underwent repeat visual function tests and fluroescein angiography. In addition about $50 \%$ of those aged 20 years and above underwent repeat visual function tests and optic disc assessment. Anyone with evidence of a deterioration in visual function was referred to the ophthalmologists, who examined them and performed fluorescein angiography unless contraindicated or refused.

\section{Angiogram Assessment}

All angiograms were read according to a structured protocol by two ophthalmologists (I.M. and O.B.). A third ophthalmologist (A.B.) then reviewed all angiograms with abnormal disc findings together with a random sample of normal angiograms. His findings correlated well with those of the other two ophthalmologists. The agreement on the presence or absence of disc leakage between ophthalmologists was good (kappa $=0.69$ for field ophthalmologists (I.M., O.B.) vs (A.B.). All observers were masked to the treatment allocation.

All angiograms with abnormal or suspicious disc findings were reviewed in a joint meeting of all three ophthalmologists and a final decision made concerning the status for the optic discs. Angiograms were then paired and decisions made concerning changes following dosing. All analyses with respect to treatment group were performed after all clinical and angiographic assessments had been completed.

\section{RESULTS}

A total of $6831(87.6 \%)$ of the registered population aged 5 years and above underwent the ophthalmic examination.

Table I. Baseline comparison of ivermectin and placebo groups with regard to age, sex and microfilarial load

\begin{tabular}{lrr}
\hline & Ivermectin & Placebo \\
\hline Age (years) & & \\
$5-14$ & $1282(37)$ & $1251(37)$ \\
$15-24$ & $624(18)$ & $621(18)$ \\
$25-34$ & $565(17)$ & $544(16)$ \\
$35-44$ & $403(12)$ & $450(13)$ \\
$45-54$ & $303(9)$ & $287(8)$ \\
$55-64$ & $146(4)$ & $161(5)$ \\
$65+$ & $97(3)$ & $97(3)$ \\
Sex & & $1694(50)$ \\
Male & $1675(49)$ & $1717(50)$ \\
Female & $1745(51)$ & $1648(50)$ \\
Microfilarial load (mf/mg) & $1742(52)$ & $1000(30)$ \\
Negative & $997(30)$ & $656(20)$ \\
-10 & $599(18)$ & $20(1)$ \\
-100 & $18(1)$ & \\
$>100$ & &
\end{tabular}

Seven hundred and twenty-six $(9.3 \%)$ were reported to be absent, $190(2.4 \%)$ refused to participate and $52(0.7 \%)$ were present but not examined, most commonly because they were ill and unable to attend for examination.

Of the 6831 individuals examined, 3420 were allocated to receive ivermectin while 3411 were allocated to receive placebo. The distributions of the ivermectin and placebo groups with regard to age and sex were very similar (Table I). There was also little difference between the two groups with regard to individual microfilarial loads (Table I).

\section{Sensitivity of the Screening Examination}

Sensitivity of the screening examination was examined in the random sample. Among those aged 20 years and above, 329 individuals in the random sample were examined. Fifty-eight (18\%) of these were found to have OND. All of these individuals had been identified by the ophthalmic nurse screening examinations, suggesting that the screening examination had a high sensitivity (95\% confidence interval 93.6-100\%).

Among those examined, a total of $613(9 \%)$ individuals were identified as having probable or definite OND. Of these, 364 (59\%) were male and 249 female. The prevalence of OND detected in the random sample was similar to that in the rest of the population (Table II). Thus there is no evidence that the nurses adopted a lower threshold for referral in the random sample because they knew the individual was going to be reviewed by the ophthalmologist.

\section{Active Optic Neuritis}

Of the 6831 persons examined, 853 (13\%) had angiography prior to dosing (Table III). Six individuals, recorded as having pink discs in one or both eyes, did not undergo angiography. In only one of these individuals were the discs thought to be pathological by the ophthalmologists. This 50-year-old woman had typical clinical signs of florid bilateral optic neuritis and refused all but the most cursory of ophthalmic examinations. She also refused a follow-up examination 7-14 days after dosing.

Sixteen cases of bilateral optic neuritis and 21 of unilateral optic neuritis were confirmed on angiography, giving a total of 37 cases (prevalence $=0.5 \%$ ). The distribution by age and sex of these 37 cases of optic neuritis is shown in Fig. 1. The median age of the cases was 42 years (range 15-67 years). There were 22 males and 15 females affected.

\section{7-14 Day Follow-up}

The ophthalmic nurses re-examined 1956 individuals

Table II. Distribution of optic nerve disease among teh population aged 20 years and above within and outside the random sample

\begin{tabular}{lrr}
\hline & \multicolumn{3}{c}{ Random sample } \\
\cline { 2 - 4 } Optic nerve disease & \multicolumn{1}{c}{ No $(\%)$} & \multicolumn{1}{c}{ Yes $(\%)$} \\
\hline No & $2772(84)$ & $271(82)$ \\
Yes & $542(16)$ & $58(18)$ \\
Total & $3314(100)$ & $329(100)$ \\
\hline
\end{tabular}

Chi-squared $=0.6(p=0.4)$. 
Table III. Examinations performed prior to dosing and prevalence of optic neuritis by treatment group

\begin{tabular}{lrrr}
\hline & & & $\begin{array}{c}\text { Not } \\
\text { treated }\end{array}$ \\
\hline Population eligible for examination & 3131 & 3139 & 1530 \\
No. undergoing screening examination & 3130 & 3138 & 563 \\
No. examined by ophthalmologist & 525 & 545 & 80 \\
No. undergoing fluorescein angiography & 419 & 415 & 19 \\
No. of cases of optic neuritis & 19 & 17 & 1 \\
\hline
\end{tabular}

7-14 days after dosing (Table IV). Of the 706 follow-up examinations performed by the ophthalmologists at 7-14 days after dosing 668 were on persons who had been examined by the ophthalmologists prior to dosing. This represented 58\% follow-up of the 1150 individuals who were examined by the ophthalmologists prior to dosing. The remaining 38 were individuals whose test results suggested they had suffered a deterioration in visual functionbetween the two examinations. Of these, 20 were in the ivermectin group and 18 in the placebo group.

\section{Friedmann Fields}

A total of 642 individuals had Freidmann visual field analysis before and 7-14 days after dosing. Each quadrant of the right and left fields was recorded as having a defect if 4 or more target points showed diminished sensitivity or 3 or more target points were missed in that quadrant.

The results after 7-14 days are shown in Table $\mathrm{V}$ by treatment group. As can be seen there was no difference between ivermectin and placebo treated groups. In view of the larger number of improvements compared with deteriorations further analysis was not performed since the learning effect with the test would mask any smaller change of visual field between groups.

\section{Optic Neuritis}

A total of 688 angiograms were completed at follow-up with 643 individuals having paired angiograms. Four individuals were identified as having developed active optic neuritis during the period since dosing; 2 of these had pre-

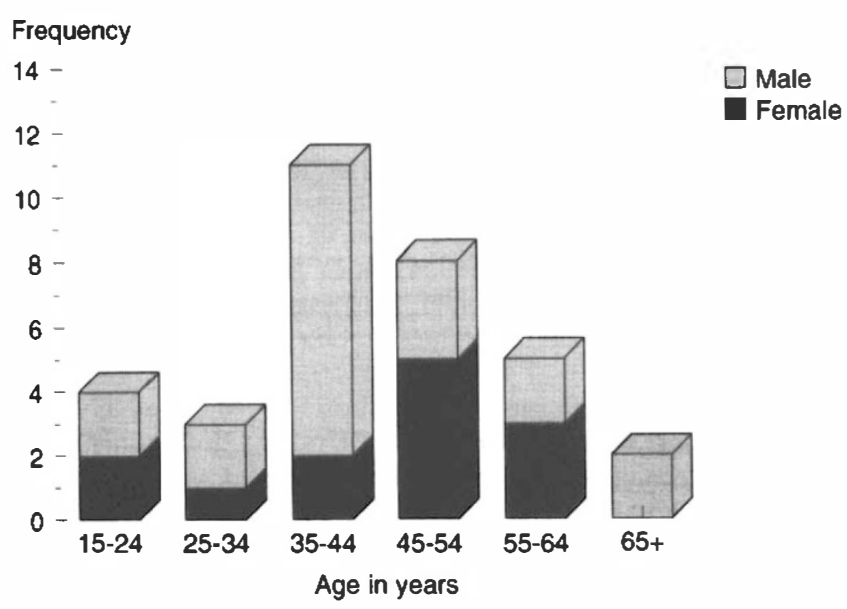

Fig. 1. Age and sex distribution of active optic neuritis in rural communities mesoendemic for onchocerciasis, northern Nigeria.
Table IV. Examinations performed 7-14 days after dosing and prevalence of optic neuritis by treatment group

\begin{tabular}{|c|c|c|c|}
\hline & Ivermectin & Placebo & $\begin{array}{l}\text { Not } \\
\text { treated }\end{array}$ \\
\hline $\begin{array}{l}\text { Re-examined by ophthalmic nurses after } \\
\text { dosing }\end{array}$ & 962 & 928 & 66 \\
\hline Total no. examined by ophthalmologist & 347 & 347 & 12 \\
\hline No. undergoing fluorescein angiography & 337 & 335 & 11 \\
\hline No. of cases of optic neuritis & 18 & 21 & 1 \\
\hline
\end{tabular}

viously been examined by the ophthalmologists and 2 had passed the screening tests prior to dosing and failed the follow-up examinations. In addition 1 individual had an increase in severity of optic neuritis in one eye. Four of these cases were placebo treated and one ivermectin treated (Table VI).

There were two people in whom the optic neuritis improved over the 7-14 day period as judged by a reduction of leakage on fluorescence angiography and improvement in visual field test results. One of these received ivermectin and the other placebo (Table II).

There were no paired field data on 3 of those patients that deteriorated; in the remaining 3, there were 2 who showed deterioration in their visual field results and the final individual passed all visual field tests on both occasions.

\section{DISCUSSION}

The prevalence of OND in mesoendemic onchocercal communities is poorly reported. Anderson et al. ${ }^{11}$ looked at those aged 5 years or more and reported a prevalence of $1.7 \%$ in hyperendemic savannah populations and $2.9 \%$ in hyperendemic rain forest populations in the Cameroon. Later, however, the same authors reported an incidence of $2.5 \%$ and $3.1 \%$ respectively in the same populations over a 5 -year period. ${ }^{12}$ With such a high incidence rate one would expect a higher prevalence than that reported. This could be explained by either a considerable mortality associated

Table V. Distribution of changes in Friedmann visual field assessment between initial and follow-up examinations by treatment group

\begin{tabular}{lccc}
\hline Friedmann field & Ivermectin & Placebo & Not treated \\
\hline No change & 135 & 144 & 5 \\
Improvement in one eye, & 10 & 14 & 1 \\
$\quad$ deterioration in one eye & 46 & 48 & 1 \\
Improvement in one eye & 54 & 53 & 2 \\
Improvement in two eyes & 41 & 32 & 1 \\
Deterioration in one eye & 27 & 27 & 1 \\
Deterioration in two eyes & 313 & 318 & 11 \\
No. examined &
\end{tabular}

Chi-squared $=2.1(p=0.8)$ for ivermectin versus placebo.

Table VI. Distribution of changes in optic neuritis status between initial and follow-up examinations by treatment group

\begin{tabular}{lccc}
\hline & \multicolumn{3}{c}{ Optic neuritis } \\
\cline { 2 - 4 } & New case & Worse & Better \\
\hline Ivermectin & 1 & 0 & 1 \\
Placebo & 4 & 1 & 1 \\
\hline
\end{tabular}


Table VII. Previous reports of effect of ivermectin on onchocercal optic neuritis

\begin{tabular}{|c|c|c|c|c|c|c|}
\hline Trial & Country & Population & Treatment & Optic atrophy & Optic neuritis & Change \\
\hline Newland et al. ${ }^{15}$ & Liberia & 200 & Placebo, 100,150 , and $200 \mu \mathrm{g} / \mathrm{kg}$ ivermectin & 8 & 1 & None \\
\hline Rothova et al. ${ }^{16}$ & Sierra Leone & 48 & Ivermectin $(150 \mu \mathrm{g} / \mathrm{kg})$ & 13 & None & None \\
\hline Greene et al. ${ }^{18}$ & Liberia & 30 & Placebo, ivermectin $(200 \mu \mathrm{g} / \mathrm{kg})$ and DEC & None & None & None \\
\hline
\end{tabular}

DEC, diethylcarbamazine citrate.

with the development of OND or an initial underestimate of the true prevalence of the finding. McMahon et al. ${ }^{13}$ reported a prevalence of $13.8 \%$ for optic atrophy in hyperendemic rain forest populations in Sierra Leone. Rates in the male population were approximately twice as high as those in females. Our finding of 537 cases (7.9\%) in mesoendemic populations is closer to McMahon's results. It should be remembered that our prevalence is for mesoendemic savannah onchocerciasis whilst that of McMahon is for hyperendemic rain-forest onchocerciasis.

To our knowledge this is the first report of the prevalence of active optic neuritis in a population mesoendemic for onchocerciasis. In parallel with our findings for optic atrophy, ${ }^{14}$ more males than females had active optic neuritis. The highest number of male cases was in the age group 35-44 years whereas the greatest number of female cases was in the age group 45-54 years which parallels an earlier rise in males of the age-specific prevalence of optic atrophy. Our finding of more optic nerve pathology in males than females matches the findings of other groups of higher prevalences of OND and blindness amongst males. ${ }^{10,12}$

Past reports of the effect of ivermectin therapy on optic neuritis have involved small groups of selected patients and low numbers with OND (Table VII). None of these studies has suggested an important short-term effect of ivermectin on OND; however, their power to detect such a difference was limited by the small numbers screened and followed-up. The trial reported by Bird et al. ${ }^{6}$ three-quarters of individuals with onchocerciasis had acute onset or exacerbation of optic neuritis after taking DEC. With the level of repeated angiography achieved (approximately 300 in each group) and the incidence of active optic neuritis observed in the placebo group, this trial had a power of $90 \%$ to detect a 5-fold (or greater) increase in the incidence of active optic neuritis associated with taking ivermectin. The impact of DEC treatment reported by Bird et al. was considerably greater than this with an incidence rate of $50 \%$ associated with treatment with DEC in their specially selected sample with OND. Although we cannot exclude the possibility that ivermectin precipitated or exacerbated optic neuritis in a small number of individuals in our population, our data show no evidence of such an effect. Indeed, in our population ivermectin appears, if anything, to be associated with a lowering in risk. It must, however, be borne in mind that microfilarial loads were lower in our population than in the population studied by Bird et al. Extrapolation of our findings to populations with much heavier infections is problematic.
The field work was carried out within the Kaduna Collaboration for Research on Onchocerciasis and received financial support from the UNDP/World Bank/WHO Special Programme for Research and Training in Tropical Diseases ID 890456, the Leverhulme Trust, and the Royal Commonwealth Society for the Blind. The untiring work of the whole field team, the logistical support team and the data processing group is gratefully acknowledged.

Key words: Ivermectin, Nigeria, Onchocerciasis, Optic neuritis.

\section{REFERENCES}

1. WHO Expert Committee on Onchocerciasis. Third report. WHO Technical Report Series no. 752, 1987.

2. Prost A. The burden of blindness in adult males in the savannah villages of West Africa exposed to onchocerciasis. Trans R Soc Trop Med Hyg 1986;80:525-7.

3. Mazzotti L. Presencia de microfilarias de Onchocerca volv'ulus en el liquido cefalorraquideo de enfermos tratados con hetrazan. Rev Inst Salubr Enferm Trop (Mex) 1959; 19:1-5.

4. Anderson J, Fuglsang H, Marshal TF de C. Effects of diethylcarbamazine on ocular onchocerciasis. Tropenmed Parasitol 1976;27:263-78.

5. Anderson J, Fuglsang H. Further studies on the treatment of ocular onchocerciasis with diethyl-carbamazine and suramin. Br J Ophthalmol 1978;62:450-7.

6. Bird AC, Hadi El Sheikh, Anderson J, Fuglsang H. Changes in visual function and in the posterior segment of the eye during therapy of onchocerciasis with diethylcarbamazine citrate. Br J Ophthalmol 1980;64:191-200.

7. Aziz MA, Diallo S, Diop IM, Lariviere M, Porta M. Efficacy and tolerance of ivermectin in human onchocerciasis. Lancet 1982;2:171-3.

8. Prod'hon J, Boussinesq M, Fobi G, Prud'hom JM, Enyong P, Lafleur C, Quillevere D. Lutte contre l'onchocercose par ivermectine: resultats d'une campagne de masse au NordCameroun. Bull Organ Mondiale Sante 1991;69:443-50.

9. Dadzie KY, Awadzi K, Bird AC, Schulz-Key H. Ophthalmological results from a placebo controlled comparative 3 -dose ivermectin study in the treatment of onchocerciasis. Trop Med Parasitol 1989;40:355-60.

10. Abiose A, Murdoch I, Babalola O, Cousens S, Liman I, Onyema J, Jones B. The distribution and aetiology of blindness and visual impairment in mesoendemic onchocercal communities, Kaduna State, Nigeria. Br J Ophthalmol (in press).

11. Anderson J, Fuglesang H, Hamilton PJS, Marshall TF de C. Studies on onchocerciasis in the United Cameroon Republic. I. Comparison of populations with and without Onchocerca volvulus. Trans R Soc Trop Med Hyg 1974;68: 190-208.

12. Anderson J, Fuglsang H, Marchall TF de C. Studies on onchoceriasis in the United Cameroon Republic. III. A four year follow-up of 6 rain-forest and 6 Sudan-savannah villages. Trans R Soc Trop Med Hyg 1976;70:362-73.

13. McMahon JE, Sowa SCI, Maude GYH, Hudson CM, Kirk- 
wood BR. Epidemiological studies of onchocerciasis in forest villages of Sierra Leone. Trop Med Parasitol 1988;39: 251-9.

14. Abiose A, Jones B, Cousens S, Murdoch I, Cassels-Brown A, Babalola O, Alexander N, et al. A randomised, controlled trial of ivermectin for onchocerciasis: evidence for a reduction in incidence of optic nerve disease. Lancet 1993;1: $130-4$.

15. Newland HR, White AT, Greene BM, D'Anna S, KeyvanLarijani E, Aziz MA, et al. Effect of single-dose ivermectin therapy on human Onchocerca volvulus infection with onchocercal ocular involvement. Br J Ophthalmol 1988;72: $561-9$
16. Rothova A, Van der Lelij A, Stilma JS, Klassen-Broekema $\mathrm{N}$, Wilson WR, Barbe RF. Ocular involvement in patients with onchocerciasis after repeated treatment with ivermectin. Am J Ophthalmol 1990;110:6-16.

17. Dadzie KY, Bird AC, Awadzi K, Schulz-Key H, Gilles HM, Aziz MA. Ocular findings in a double-blind study of ivermectin versus diethylcarbamazine versus placebo in the treatment on onchocerciasis. Br J Ophthalmol 1987;71: 78-85.

18. Greene BM, Taylor HR, Cupp EW, Murphy RP, White AT, Aziz MA, et al. Comparison of ivermectin and diethylcarbamazine in the treatment of onchocerciasis. $\mathrm{N}$ Engl $\mathrm{J}$ Med 1985;313:133-8. 